\title{
Metal-Insulator Transitions at Surfaces
}

\author{
Michael Potthoff \\ Lehrstuhl Festkörpertheorie, Institut für Physik, Humboldt-Universität zu Berlin, \\ Germany
}

\begin{abstract}
Various types of metal-insulator transitions are discussed to find conditions for which an ideal surface of a bulk insulator is metallic. It is argued that for the correlation-driven Mott metal-insulator transition the surface phase diagram should be expected to have the same topology as the phase diagram for magnetic order at surfaces: The corresponding linearized mean-field descriptions, a simplified dynamical mean-field theory of the Hubbard model and the Weiss mean-field theory for the Ising model, are found to be formally equivalent. A new kind of surface state appears in the low-energy part of the one-particle excitation spectrum as a precursor effect of the Mott transition.
\end{abstract}

The Mott metal-insulator transition at a crystal surface is a subject that touches different areas in solid-state theory which are usually treated as being disjoined: many-body theory of the correlation-driven metal-insulator transition, the general theory of surface phase transitions, and the theory of electronic surface states. It is the intention of the present paper to show that a corresponding combination of different concepts can be fruitful and allows for some new theoretical predictions.

\section{Surface Phase Transitions}

The large variety of novel and interesting phenomena in surface physics is closely related with the occurrence of surface phase transitions. As has been pointed out by Mills [1], the surface of a system may undergo a phase transition at a critical temperature $T_{\mathrm{c}, \mathrm{s}}$ being different from the bulk critical temperature $T_{\mathrm{c}}$, i.e. the surface may undergo its own phase transition. Critical exponents, for example, can be defined and determined which are specific for the transition at the surface and which cannot be fully reduced to the bulk critical exponents [2:3]. Different kinds of surface phase transitions are conceivable and have been found, e.g. structural transitions, such as deviating geometrical order of the atoms near the surface of a single crystal (surface reconstruction), the loss of long-range crystalline order at the surface prior to a bulk melting transition (surface melting) or enrichment of one component at the surface of a solid binary alloy (surface segregation) [2. Typical examples for surface phase transitions are also found among magnetically ordered systems: For example, the (0001) surface of ferromagnetic Gd is believed to have a Curie temperature which is higher than the bulk $T_{\mathrm{C}}$ [प]. 
Different types of surface phase transitions can be described in a qualitative but consistent way by means of classical Landau theory [2, 3] or by lattice mean-field approaches which may be considered as coarse-grained realizations of the Landau theory. Especially, mean-field approaches to localizedspin models, such as the Ising or Heisenberg model, are frequently considered in this context [5]. For surface geometries there are a number of nontrivial results predicted by Landau or mean-field theory, such as temperaturedependent order-parameter profiles, which may give a surprisingly good description of experimental data (see Ref. [6], for example).

Typically, the surface undergoes the phase transition at the same temperature as in the bulk, $T_{\mathrm{c}, \mathrm{s}}=T_{\mathrm{c}}$, if the local (structural, electronic, magnetic) environment remains unchanged, while $T_{\mathrm{c}, \mathrm{s}}>T_{\mathrm{c}}$ if there is a perturbation $\Delta$ at the surface exceeding a certain critical value $\Delta_{c}$. For $\Delta>\Delta_{\mathrm{c}}$ and temperatures $T_{\mathrm{c}}<T<T_{\mathrm{c}, \mathrm{s}}$ there is an ordered $D$-1-dimensional surface coexisting with a disordered $D$-dimensional bulk. More complicated phase diagrams are obtained in the case of multi-critical behavior, e.g. when the long-range order at the surface has a character different from the long-range order in the bulk (surface reconstruction, anti-ferromagnetic surface of a ferromagnetic bulk, etc). The Landau $T-\Delta$ phase diagram should be qualitatively correct whenever the $D-1$-dimensional system can support independent order [2, 7 ].

\section{Metal-Insulator Transitions}

The concept of a surface phase transition and the corresponding Landau theory seems to extend straightforwardly to a certain kind of metal-insulator transitions, namely those which accompany an order-disorder thermodynamic phase transition (see Ref. [8] for an overview). The thermodynamic phase transition will be considered at the $T=0$ quantum-critical point as due to ubiquitous thermal activation processes, a strict definition of a metalinsulator transition is possible at zero temperature only. It is well known that the formation of an ordered state may result in a gap for charge excitations as, for example, in the Peierls transition or in the transition to an antiferromagnetic state: Consider the typical example of a bipartite lattice and two-sublattice long-range order causing a doubling of the unit cell. For a nondegenerate band the splitting at the boundary of the new Brillouin zone will lead to a gap and, in the case of half-filling, to an insulating ground state.

Now, for sublattice order at the surface of a disordered bulk one would have the (naive) expectation that an insulating surface could coexist with a metallic bulk. This, however, is clearly impossible as a finite bulk density of states at the Fermi energy will always induce a non-zero, though possibly low density of states in the surface region. Likewise, the reverse scenario is impossible either: Namely, to realize a metallic surface phase of a bulk insulator caused by a thermodynamic phase transition, a disordered surface would have to coexist with an ordered bulk which, in general, is forbidden by 
strict arguments [2] (though a magnetic "dead-layer" scenario is found under somewhat exotic circumstances in a $D=2 q$-state surface Potts model [9]).

Besides the thermodynamic transitions, there is a second important class of metal-insulator transitions, namely quantum-phase transitions [8]. Essentially these take place at $T=0$ only and are not associated with any symmetry breaking. Important examples are the transition from a metal to a normal band insulator and the Mott-Hubbard transition from a metallic Fermi liquid to a Mott insulator. While the former can be understood within an independent-electron model, correlation effects are constitutive for the latter. Referring to a quantum-phase metal-insulator transition, it is very well feasible that the surface of a bulk insulator is metallic.

\section{Surface States}

Fig. 11 shows a possible electron density of states for this situation. To have a metallic surface of an insulator, the density of states at the Fermi energy $E_{\mathrm{F}}$ must be finite at the surface while, with respect to the bulk states, $E_{\mathrm{F}}$ should lie within a band gap. Note that this necessarily implies the existence of a partially filled surface state: The appearance of a surface state at the Fermi energy is crucial to get a metallic surface of an insulator.

Two possible origins of electronic surface states are well known [10]: (i) Image-potential states may arise as Rydberg-like states in the long-ranged $-1 / 4 z$ image potential which is due to the polarization charge that is induced by an electron approaching a conductive surface. The electron can be trapped between the image-potential surface barrier and the bulk barrier due to a bulk band gap. (ii) Crystal-induced surface states originate from the mere crystal termination. For an ideal unreconstructed crystal surface a further distinction between Tamm and Shockley states is meaningful: Shockley states appear within a hybridizational band gap which may open when the boundaries of two bulk bands have crossed as a function of decreasing lattice constant. Tamm-like surface states are due to the surface change of the one-electron potential and always lie near the bulk band from which they originate.

In fact, a crystal-induced surface state may give rise to a metallic surface phase as is demonstrated by the following two examples: Due the reconstruction of the $\mathrm{Si}(111)-(7 \times 7)$ surface there is a partially occupied surface state which is consistent with the observed metallic behavior for this surface (cf. the discussion in Ref. 10 |). A surface insulator-to-metal transition has been predicted for the ferromagnetic insulator $\mathrm{EuO}$ [11]: For temperatures below $T_{\mathrm{C}}=69 \mathrm{~K}$ and decreasing, the majority $5 d$ conduction band shifts towards the occupied $4 f-\uparrow$ bands thereby reducing the insulating gap. This so-called red shift is transmitted to an unoccupied Tamm state which is predicted to split off at the (100) surface from the lower edge of the conduction band. The energy difference between the surface state and the majority bulk band edge together with the $T$-dependent red shift is just sufficient to bridge the gap. 


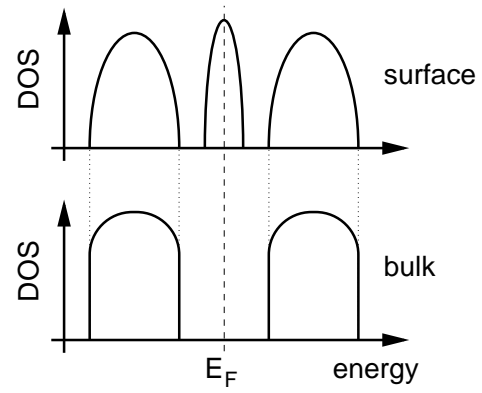

Fig. 1. Bulk and the surface density of states (schematic) in case of a metallic surface and an insulating bulk. $E_{\mathrm{F}}$ : Fermi energy.

Therefore, the surface state should become populated for low $T$ which would imply a transition to a (half-)metallic state at the surface.

In any case the concept of a crystal-induced surface state is based on a model of effectively independent electrons. It is the detailed form of the oneelectron potential that determines the energy position (lateral dispersion) of a surface state in the band structure. Hence, it is obvious that a surface state is not normally pinned to $E_{\mathrm{F}}$ (chemical potential); one may conclude that for a surface of a band insulator there is no a priori reason for a surface state to be partially filled.

\section{Mott Transition}

The question is whether or not a situation is conceivable where the appearance of the surface state is necessarily connected with the metal-insulator transition, i.e. where the surface state necessarily appears at $E_{\mathrm{F}}$. It is now clear that to this end one has to look for an electron-correlation effect.

Consider electrons in a narrow non-degenerate band interacting with each other via an on-site Coulomb repulsion $U$ as described by the Hubbard model [12. Any symmetry-broken phases will be excluded from the discussion. At half-filling $n=1$ and for strong $U$, the system is then a paramagnetic insulator. The $\mathbf{k}$-integrated one-electron excitation spectrum (DOS) of this so-called Mott-Hubbard insulator [8,13 has the same form as shown in the lower part of Fig. 1 where now the two peaks have to be interpreted as the lower and the upper Hubbard band separated by an energy of the order of $U$.

On the other hand, for $U=0$ and for the weakly interacting case, the system is a normal Fermi liquid. At an intermediate interaction strength $U_{\mathrm{c}}$ of the order of the width $W$ of the non-interacting DOS one thus expects a metal-insulator transition. This Mott transition is a prime example for a quantum phase transition at $T=0$ which results from the competition between the electrons' kinetic energy $\sim W$ which tends to delocalize the electrons and their potential energy $\sim U$ which tends to localize them. 
The Mott transition is a true many-body effect that cannot be explained by a simple perturbational approach. Even within the framework of simplified model Hamiltonians, as the Hubbard model, an ultimate theory of the Mott transition is still missing [8]. A decisive step forward, however, has been made in the last decade with the development of dynamical mean-field theory (DMFT) and its application to the Mott transition (see Sec. 5 , for a review see Ref. [14, recent results can be found in Ref. [15]).

Within the DMFT one finds that the transition at $U=U_{\mathrm{c}}$ is characterized by a diverging effective mass $m^{*} \rightarrow \infty$ or a vanishing quasi-particle weight $z \propto\left(m^{*}\right)^{-1} \rightarrow 0$, respectively. For $U<U_{\mathrm{c}}$ but close to the critical point, the DOS has a three-peak structure, consisting of the two well-developed Hubbard bands as well as a narrow quasi-particle resonance at the Fermi energy with weight $z$ - the DOS has the same form as the "surface DOS" shown by the upper part of Fig. 1.

It is conceivable that Fig. 1 describes a situation where the bulk of the system is a Mott-Hubbard insulator while the surface is in a metallic Fermiliquid state. The quasi-particle resonance would then be a surface state (oneelectron surface excitation) with a layer-dependent weight $z_{\alpha}$ decreasing exponentially with increasing distance from the surface. This surface state necessarily appears at the Fermi energy as it corresponds to low-energy excitations well known from quantum impurity systems (Kondo effect). The question is for which circumstances this coexistence of the Mott-Hubbard insulator and the metallic Fermi liquid can be realized.

\section{Mean-Field Approach}

As it is by no means obvious how to construct a (continuum) Landau theory for this problem, the method of choice is to formulate and evaluate a meanfield theory for an appropriate discrete lattice model. While for a magnetic phase transition one can resort to effective spin models such as the Ising model without any detailed knowledge of the electronic structure, the Hubbard model as the minimum model to describe the Mott transition includes spin as well as charge degrees of freedom and is thus much more complicated. Likewise it is much more complicated to find a proper mean-field theory. For example, Hartree-Fock theory, weak- and strong-coupling perturbational approaches or decoupling approximations for the Hubbard model are clearly inferior compared with the Weiss mean-field theory for the Ising model. The latter is non-perturbative, thermodynamically consistent and free of unphysical singularities in the entire parameter space. Since the Weiss theory becomes exact in the non-trivial limit of infinite spatial dimensions $D$ [16], this may serve as a simple and precise characterization of what is a proper mean-field theory in general. One may therefore hope that the same limit will lead to a powerful mean-field approach in the case of the Hubbard model, too. 

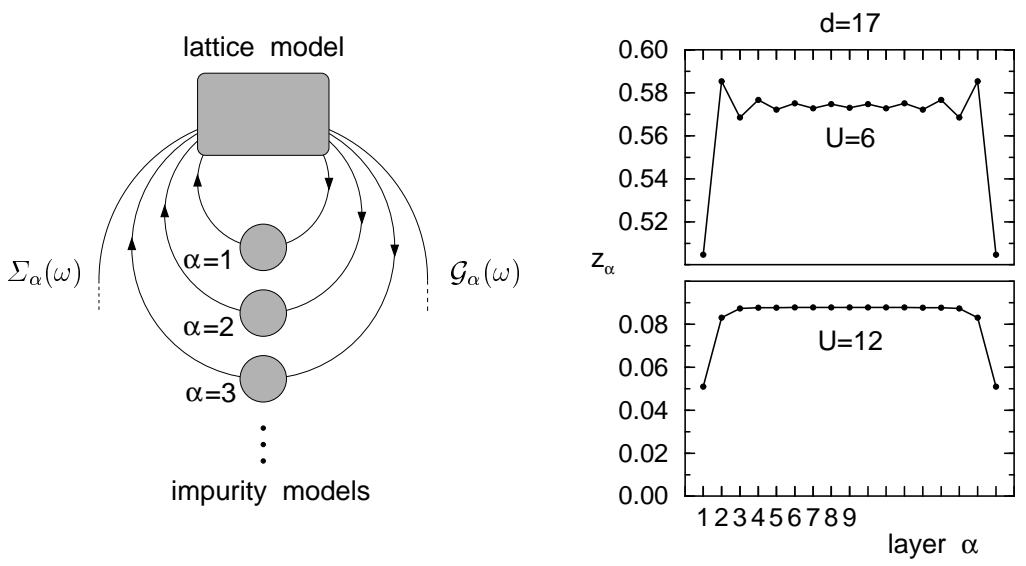

Fig. 2. Left: DMFT self-consistency cycle for the Hubbard model on a semi-infinite lattice with layer index $\alpha=1, \ldots, d(d \rightarrow \infty)$. See text for discussion. Right: Layerdependent quasi-particle weight $z_{\alpha}$ for a simple-cubic film of $d=17$ layers with (100) surfaces. DMFT results for uniform nearest-neighbor hopping $t=1$, halffilling $n=1, T=0$ and $U$ as indicated.

That the $D=\infty$ limit for a lattice fermion model is well-defined and non-trivial in fact, has been proven in the pioneering work of Metzner and Vollhardt [17]. Crucial is a proper scaling of the hopping $t \propto 1 / \sqrt{D}$ to keep the dynamic balance between kinetic and potential energy. To convert the abstract definition of a proper mean-field theory into a useful concept for practical calculations, it has been important to realize that the $D=\infty$ Hubbard model can be mapped onto the single-impurity Anderson model (SIAM) as now one can profit from various methods available for impurity problems. This observation has been made by Georges, Kotliar and Jarrell 18. 19. The mapping is a self-consistent one which means that the parameters of SIAM depend on the one-particle Green function of the Hubbard model.

To study surface effects one has to consider a variant of the original Hubbard model. Using standard notations the Hamiltonian reads:

$$
H=\sum_{i_{\|} j_{\|} \alpha \beta \sigma} t_{i_{\|} \alpha, j_{\|} \beta} c_{i_{\|} \alpha \sigma}^{\dagger} c_{j_{\|} \beta \sigma}+\frac{U}{2} \sum_{i_{\|} \alpha \sigma} n_{i_{\|} \alpha \sigma} n_{i_{\|} \alpha-\sigma} .
$$

Here $i_{\|}$labels the sites within a layer $\alpha$ parallel to the surface. $\alpha=1$ corresponds to the top surface layer. The model (1D) differs from the original Hubbard model by the mere existence of the surface: The sole effect of the surface is to terminate the bulk. For any numerical calculation one has to assume a finite number of layers, $\alpha=1, \ldots, d$, i.e. a film geometry, and to check the convergence of the results for large $d \rightarrow \infty$.

The generalization of DMFT for surface geometries has been developed by Potthoff and Nolting [20]. Again, the limit $D=\infty$ may serve as a guide to 
construct a powerful mean-field theory. Assuming uniform hopping parameters, $t_{i_{\|} \alpha, j_{\|} \beta}=t$ between nearest neighbors $\left(i_{\|}, \alpha\right)$ and $\left(j_{\|}, \beta\right)$, and using the same scaling $t \propto 1 / \sqrt{D}$, the model itself as well as surface effects remain non-trivial for $D \rightarrow \infty$. As the different layers parallel to the surface must be treated as being inequivalent, the mapping procedure, however, becomes more complicated (see Fig. 2, left). The original many-body problem for a semi-infinite lattice with layers $\alpha=1,2, \ldots, d$ (with $d \rightarrow \infty$ ) is selfconsistently mapped onto a set of impurity problems labeled by the same index $\alpha=1,2, \ldots, d$. Each SIAM can be treated independently to calculate the impurity self-energy $\Sigma_{\alpha}(\omega)$. There is, however, an indirect coupling which is mediated by the self-consistency cycle: Via the Dyson equation of the lattice model, the on-site Green function $G_{\alpha}(\omega)$ for a given layer $\alpha$ depends on all layer-dependent self-energies. The free Green function $\mathcal{G}_{\alpha}(\omega)$ of the $\alpha$ th SIAM which determines its one-particle parameters is then obtained from the DMFT self-consistency condition: $\mathcal{G}_{\alpha}(\omega)^{-1}=G_{\alpha}(\omega)^{-1}+\Sigma_{\alpha}(\omega)$.

Fig. 2 (right) shows the quasi-particle weight $z_{\alpha}=\left(1-\Sigma_{\alpha}^{\prime}(0)\right)^{-1}$ as obtained from the DMFT using a standard ("exact diagonalization") method 21 to treat the different impurity problems. The critical interaction $U_{\mathrm{c}}(d)$ of the $d=17$ layer $\mathrm{sc}(100)$ film lies close to the bulk critical interaction $U_{\mathrm{c}, \text { bulk }} \approx 16$ ( $W=12$ is the width of the free bulk DOS). In the metallic phase for $U<U_{\mathrm{c}}(d)$ there is a quasi-particle resonance in the interacting local density of states for each layer with a finite weight $z_{\alpha}$. As can be seen in the figure, the weight has a pronounced layer dependence. While for small $U$ the profile has an oscillating character, it becomes monotonous for interaction strengths close to the transition. This is a typical result which is observed for films with different surface geometries and indicates a universal behavior of the critical profile for $U \rightarrow U_{\mathrm{c}}(d)$. For both, $U=6$ and $U=12$, the surface quasi-particle weight $z_{1}$ is considerably lower than the bulk quasi-particle weight $z$ at the film center. This result is plausible: Due to the reduced coordination number at the surface $q_{1}<q$, the variance $\Delta_{1}=q_{1} t^{2}$ of the surface-layer DOS is reduced which implies the "effective" interaction $U / \sqrt{\Delta_{1}}$ to be stronger at the surface compared with the bulk. In this respect the surface is "closer" to the insulating phase. Yet, for $U \rightarrow U_{\mathrm{c}}(d)$ all $z_{\alpha}$ vanish simultaneously and there is no surface transition.

\section{Critical Regime}

For systematic investigations of films with different (large) thicknesses, with different surface geometries and for different model parameters, a numerically exact evaluation of the DMFT requires an effort which is out of scale. Fortunately, a simplified treatment of the mean-field equations is possible at $T=0$ for parameters close to the critical point as has been pointed out by Bulla and Potthoff [22]. This "linearized DMFT" (L-DMFT) is based on two plausible assumptions for the critical regime $U \rightarrow U_{\mathrm{c}}$ : (i) The effect of the two 


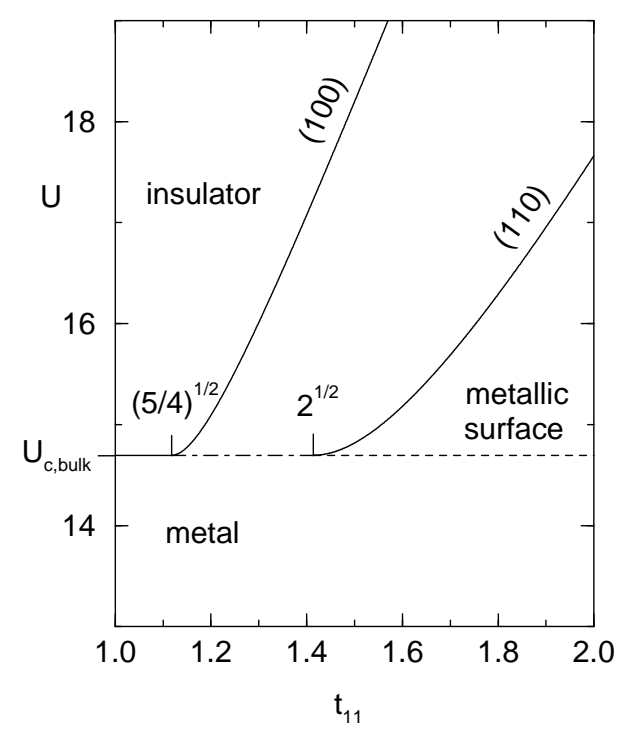

Fig. 3. Phase diagram for the Mott transition at the (100) and (110) surface of the sc lattice. $t_{11}(\geq t=1)$ : modified hopping within the surface layer $\alpha=1$. $U_{\mathrm{c}, \text { bulk }}$ : bulk critical interaction.

Hubbard bands on the quasi-particle resonance can be disregarded and the resonance basically reproduces itself in the DMFT self-consistency cycle. (ii) The resonance has no internal structure and can be described by a one-pole approximation. Although these assumptions are approximate, the L-DMFT has successfully passed a number of tests which have been performed by comparing with the full theory and which show that the L-DMFT is well qualified to give quantitative estimates for critical interactions and critical profiles as well as the correct topology of phase diagrams [22,20,23].

Within the L-DMFT the mean-field equations reduce to algebraic equations for $z_{\alpha}$ which involve the electronic model parameters and the system geometry. For example, in the case of a surface geometry with $q_{\|}$nearest neighbors within a layer, $q_{\perp}$ nearest neighbors within each of the adjacent layers and with uniform hopping $t$ and interaction $U$ except for the hopping $t_{11} \neq t$ within the surface layer $\alpha=1$, the mean-field equations read:

$$
z_{1}=\frac{36}{U^{2}}\left(q_{\|} t_{11}^{2} z_{1}+q_{\perp} t^{2} z_{2}\right), \quad z_{\alpha}=\frac{36 t^{2}}{U^{2}}\left(q_{\|} z_{\alpha}+q_{\perp} z_{\alpha+1}+q_{\perp} z_{\alpha-1}\right)
$$

for $\alpha=2,3, \ldots, \infty$. Equations of this type can be solved analytically or by simple numerical means.

Fig. 3 shows the phase diagram for the Mott transition at two low-index surfaces of a semi-infinite $D=3$ simple cubic lattice as obtained within the L-DMFT. For the unperturbed surface $\left(t_{11}=t\right)$ there is a single metal- 


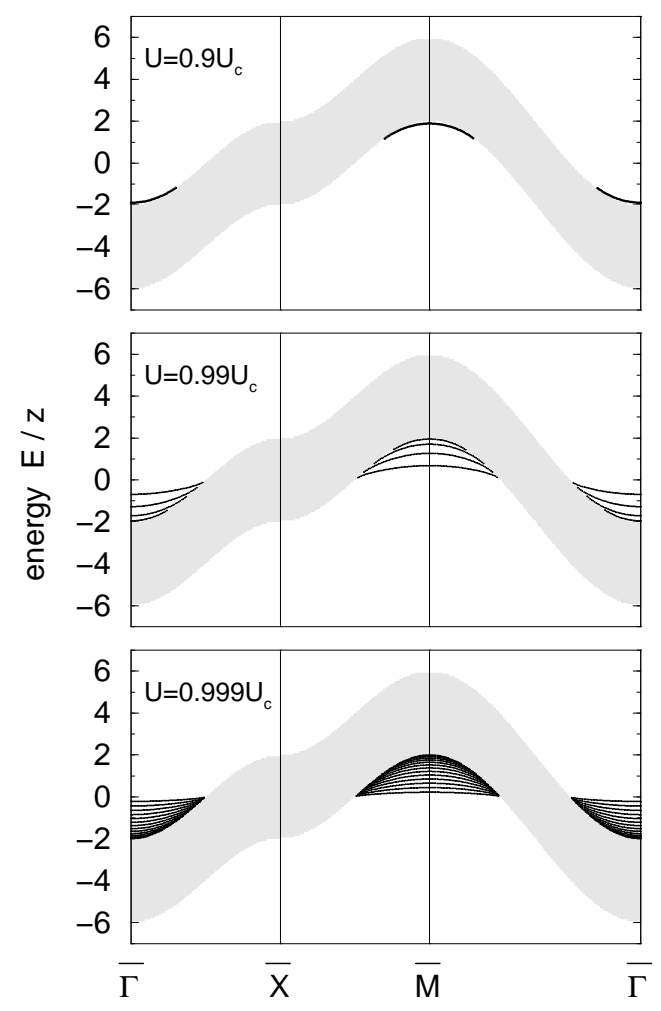

Fig. 4. Surface states (lines) in the low-energy part of the one-electron excitation spectrum and bulk continuum (grey region) in the $D=2$ Brillouin zone for the

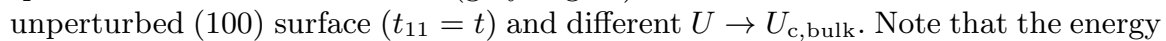
scale is normalized with respect to the bulk quasi-particle weight $z$ with $z \rightarrow 0$ for $U \rightarrow U_{\text {c,bulk }}$.

insulator transition at the bulk critical interaction ("ordinary transition"). Within the L-DMFT $U_{\text {c,bulk }}=6 t \sqrt{q_{\|}+2 q_{\perp}}=6 \sqrt{6}$. An enhancement of the surface hopping $t_{11}>t$ which exceeds a certain critical value, leads to two critical interactions: At $U=U_{\mathrm{c}, \text { bulk }}$ there is the transition of the bulk of the system irrespective of the state of the surface ("extraordinary transition"). The surface undergoes its own phase transition to the insulating state at a second critical interaction $U_{c, \text { surf }}>U_{\text {c,bulk ( }}$ (surface transition"). The critical perturbation $t_{11, c}$ depends on the surface geometry. Multi-critical behavior is found for $t_{11}=t_{11, \mathrm{c}}$ and $U=U_{\mathrm{c}, \text { surf }}=U_{\mathrm{c}, \text { bulk }}$ ("special transition").

For $U_{\mathrm{c} \text {,bulk }}<U<U_{\mathrm{c} \text {,surf }}$ a metallic surface is coexisting with an insulating bulk. As is demonstrated by Fig. 1 1 this implies the existence of a surface state. Fig. 1 shows that even for the unperturbed surface $\left(t_{11}=t\right)$ in the metallic phase close to the ordinary transition there is a surface state and in the limit $U \rightarrow U_{\mathrm{c}, \text { bulk }}$ even an infinite number of surface states. These are split 
off from the bulk continuum of coherent low-energy excitations the width of which vanishes proportional to $z \propto U_{\mathrm{c}, \text { bulk }}-U$ for $U \rightarrow U_{\mathrm{c}, \text { bulk. }}$. The critical profile of the quasi-particle weight in this limit, $z_{\alpha} \propto \alpha$, causes a strong surface perturbation of the low-energy electronic structure which drives the surface states. These surface states should be considered as a new kind of correlation-induced surface states as for the corresponding non-interacting model (with $t_{11}=t$ ) the occurrence of surface states is impossible.

Concluding, one finds a phase diagram with the same topology as predicted by the Landau theory of surface phase transitions. This analogy can even be made quantitative: Consider Weiss mean-field theory for ferromagnetic order in the Ising model on a semi-infinite lattice. For $T \rightarrow T_{\mathrm{C}}$, the mean-field equation can be linearized, and one has $m_{\alpha}=(J / 2 T)\left(q_{\|} m_{\alpha}+\right.$ $\left.q_{\perp} m_{\alpha+1}+q_{\perp} m_{\alpha-1}\right)$ which by comparing with Eq. (2) immediately yields the following correspondences: $m_{\alpha} \Leftrightarrow z_{\alpha}, J / 2 \Leftrightarrow 36 t^{2}$ and $T \Leftrightarrow U^{2}$ where $J$ is the coupling constant and $m_{\alpha}$ the layer-dependent magnetization. It is obvious that this analogy has a number of implications for the Mott transition.

For example, for a thermodynamic phase transition (analogously for the $T=0$ Mott transition) there are two critical exponents that merely involve the critical temperature (interaction strength), the "shift exponent" $\lambda_{s}$ and the "crossover exponent" $\phi$ [2,3]. They describe the trend of $T_{\mathrm{c}}\left(U_{\mathrm{c}}\right)$ for films with thickness $d \mapsto \infty$ and the trend of $T_{\mathrm{c}, \text { surf }}\left(U_{\mathrm{c}, \text { surf }}\right)$ for the semi-infinite system near the special transition, respectively. Within the Laudau theory (linearized DMFT) one finds $\lambda_{s}=2$ and $\phi=1 / 2$.

\section{Conclusions}

Among different types of metal-insulator transitions at a surface of a single crystal, the correlation-driven Mott transition from a normal metal to a paramagnetic insulator is distinguished as it offers a comparatively simple route to a metallic surface phase of an insulating bulk. Formally, this is expressed by the equivalence between the respective linearized mean-field approaches to the Mott quantum-phase transition and to the thermodynamic (magnetic) phase transition. It should be stressed that the equivalence implies that all results of the Landau theory of surface phase transitions have a unique counterpart for the surface Mott transition. This includes phase diagrams, critical profiles of the quasi-particle weight, critical exponents and other critical behavior. In this way a comprehensive and consistent mean-field picture of the characteristics of the surface Mott transition is obtained.

The author would like to thank R. Bulla and W. Nolting for discussions and collaborations. The work has been supported by the Deutsche Forschungsgemeinschaft within the Sonderforschungsbereich 290. 


\section{References}

1. D. L. Mills, Phys. Rev. B 3, 3887 (1971).

2. K. Binder, In: Phase Transitions and Critical Phenomena, Vol. 8, ed. by C. Domb and J. L. Lebowitz (Academic, London, 1983).

3. H. W. Diehl, In: Phase Transitions and Critical Phenomena, Vol. 10, ed. by C. Domb and J. L. Lebowitz (Academic, London, 1986).

4. P. A. Dowben, M. Donath and W. Nolting, editors, Magnetism and electronic correlations in local-moment systems: Rare-earth elements and compounds (World Scientific, Singapore, 1998).

5. T. Kaneyoshi, I. Tamura, and E. F. Sarmento, Phys. Rev. B 28, 6491 (1983); K. Binder and D. P. Landau, Phys. Rev. Lett. 52, 318 (1984); C. Tsallis and E. F. Sarmento, J. Phys. C 18, 2777 (1985); F. Aguilera-Granja and J. L. Morán-López, Phys. Rev. B 31, 7146 (1985); P. J. Jensen, H. Dreyssé, and K. H. Bennemann, Surf. Sci. 269/270, 627 (1992).

6. R. Pfandzelter and M. Potthoff, Phys. Rev. B 64, 140405 (2001).

7. N. D. Mermin and H. Wagner, Phys. Rev. Lett. 17, 1133 (1966).

8. F. Gebhard, The Mott Metal-Insulator Transition (Springer, Berlin, 1997).

9. R. Lipowsky, Z. Phys. B 45, 229 (1982); J. Phys. A 15, L195 (1982).

10. S. G. Davison and M. Streślicka, Basic Theory of Surface States (Clarendon, Oxford, 1992).

11. R. Schiller and W. Nolting, Phys. Rev. Lett. 86, 3847 (2001).

12. J. Hubbard, Proc. R. Soc. London A 276, 238 (1963); M. C. Gutzwiller, Phys. Rev. Lett. 10, 159 (1963); J. Kanamori, Prog. Theor. Phys. (Kyoto) 30, 275 (1963).

13. N. F. Mott, Metal-Insulator Transitions (Taylor and Francis, London, 1990).

14. A. Georges, G. Kotliar, W. Krauth, and M. J. Rozenberg, Rev. Mod. Phys. 68, 13 (1996).

15. R. Bulla, T. A. Costi, and D. Vollhardt, Phys. Rev. B 64, 045103 (2001); J. Joo and V. Oudovenko, Phys. Rev. B 64, 193102 (2001).

16. F. Englert, Phys. Rev. 129, 567 (1963).

17. W. Metzner and D. Vollhardt, Phys. Rev. Lett. 62, 324 (1989).

18. A. Georges and G. Kotliar, Phys. Rev. B 45, 6479 (1992).

19. M. Jarrell, Phys. Rev. Lett. 69, 168 (1992).

20. M. Potthoff and W. Nolting, Phys. Rev. B 59, 2549 (1999); Euro. Phys. J. B 8, 555 (1999); Phys. Rev. B 60, 7834 (1999).

21. M. Caffarel and W. Krauth, Phys. Rev. Lett. 72, 1545 (1994).

22. R. Bulla and M. Potthoff, Euro. Phys. J. B 13, 257 (2000).

23. Y. Ōno, R. Bulla, and A. C. Hewson, Euro. Phys. J. B 19, 375 (2001); Y. Ōno, R. Bulla, A. C. Hewson, and M. Potthoff, Euro. Phys. J. B 22, 283 (2001). 